\title{
Mixed Metal Oxide Nanoparticle Formulations for the Treatment of Seroma
}

Ioana Lese,* Catherine Tsai, Martin Matter, Tsering Wüthrich, Helene Sophie Scheer, Adriano Taddeo, Mihai Adrian Constantinescu, Inge Katrin Herrmann, and Radu Olariu

Cite This: https://doi.org/10.1021/acsbiomaterials.1c00283

Read Online

ACCESS |

山ll Metrics \& More

Article Recommendations

Supporting Information

ABSTRACT: Seroma formation is a well-recognized postoperative complication for many plastic and general surgical procedures. Although various tissue adhesives and substances have been used in an effort to treat seroma formation, no therapies have been established clinically. Recently, the nano-bridging phenomenon has been introduced as a promising approach to achieve tissue adhesion and strong closure of deep skin wounds in rats. The present study seeks to assess the potential of nano-bridging beyond skin wounds in a rat model of seroma. Seromas were induced in 20 Lewis rats through bilateral axillary lymphadenectomy, excision of the latissimus dorsi and cutaneous maximus muscles, and disruption of dermal lymphatics. On postoperative day (POD) 7, the seroma
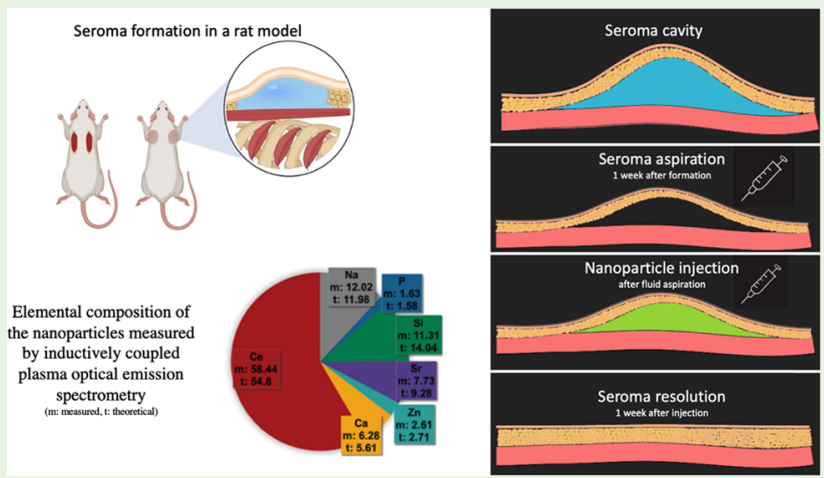
was aspirated on both sides. A bioactive nanoparticle (NP) suspension based on zinc-doped strontium-substituted bioglass/ceria nanoparticles (NP group) or fibrin glue (fibrin group) was injected into the right seroma cavity, while the left side was left untreated. On POD 14, the NP group showed complete remission (no seromas at all), while the fibrin group recorded a reduction of only 63\% in the seroma fluid volume. The NPs exerted local antiinflammatory and neo-angiogenic effects, without any detectable systemic changes. Moreover, the ceria levels recorded in the organs did not surpass the background level, indicating that the nanoparticles stayed at the site of application. This study is a promising first example demonstrating the ability of inorganic nanoparticle formulations to reduce seroma formation in a rat model, without any detectable systemic adverse effects. These results emphasize the potential of nanotechnological solutions in the therapeutic management of seroma in the clinical setting.

KEYWORDS: seroma, mixed metal oxide nanoparticles, animal model

\section{INTRODUCTION}

Seroma formation is a common yet poorly understood postoperative complication caused by the accumulation of serous fluid following the dissection of extensive skin flaps. Seromas most commonly occur following abdominoplasty (removal of excess skin and fat from the middle and lower abdomen mostly for cosmetic purposes), ${ }^{1,2}$ mastectomy (removal of breast tissue) with or without the removal of the axillary lymph nodes, ${ }^{3}$ and at the donor site after harvesting the latissimus dorsi muscle for reconstructive purposes. ${ }^{4}$ Seroma formation is a serious postoperative complication recorded in up to $79 \%$ of the patients. ${ }^{5}$ Because of its high frequency, surgeons have started to consider it a side effect rather than a complication. The therapeutic management is stressful and time-consuming for patients and physicians alike and includes prolonged hospital stays due to the required drainage, associated wound healing problems, repeated admissions to the hospital, and additional corrective surgeries. ${ }^{6}$ The exact pathogenesis of seroma formation is still under debate. Seromas are generally considered to be formed by the accumulation of the lymphatic fluid. ${ }^{7-9}$ However, several studies have also traced their origin back to surgical trauma and the resulting acute inflammatory exudation. ${ }^{10,11}$ Various studies have explored the use of tissue adhesives and substances such as methylprednisolone, ${ }^{12}$ Mytilus edulis protein, ${ }^{13}$ and lysine-derived urethane ${ }^{14}$ in attempts to reduce postoperative seroma formation. However, the aforementioned approaches failed to establish a clinical standard.

A commonly investigated pathway in the pathogenesis of seroma and the treatment thereof is the plasmin system and the components of the coagulation cascade. ${ }^{15,16}$ Specifically, the lack of fibrinogen observed on the first postoperative day (POD) has been supplemented with fibrin glue to promote

Received: March 1, 2021

Accepted: April 9, 2021 


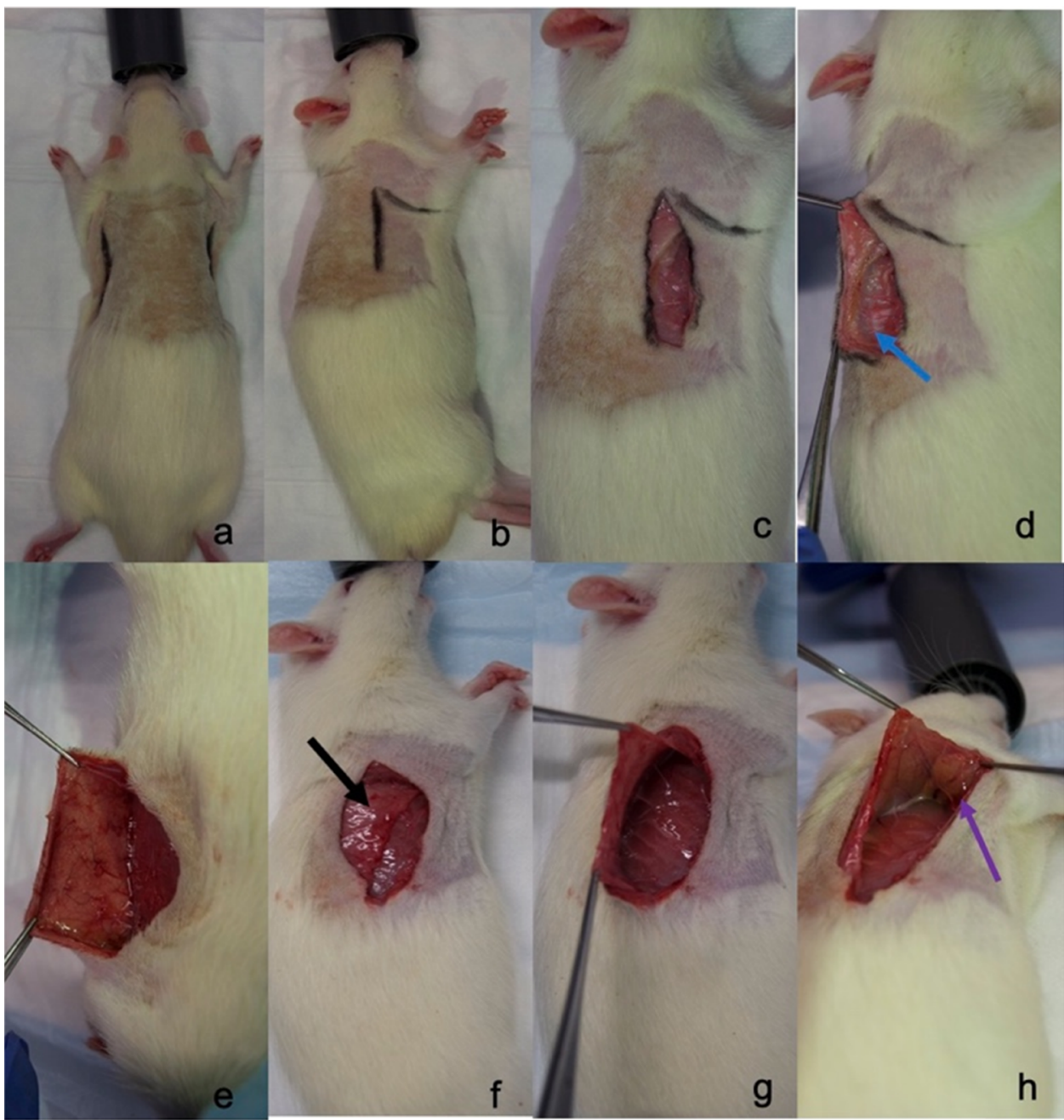

Figure 1. (a, b) Posterior axillary lines were marked on both sides. (c) $3 \mathrm{~cm}$ skin incision. (d) Identification of the cutaneous maximus muscle (blue arrow). (e) Muscle was removed $1 \mathrm{~cm}$ on each side of the incision. To disrupt the dermal lymphatics, the undersurface of the skin was scraped with a scalpel. (f) Identification of latissimus dorsi muscle (black arrow). (g) Excision of latissimus dorsi muscle. (h) Identification and removal of axillary lymph nodes (purple arrow).

coagulation. ${ }^{4,17}$ Supplementation with coagulation factors has been attempted in several studies, however, with heterogeneous results. Kulber et al. ${ }^{17}$ have previously proven the adhesive efficacy of fibrin glue in rat models; however, the fibrin adhesives ultimately failed to show any benefit in minimizing seromas in a randomized controlled trial in the clinical setting. ${ }^{4}$ The combination of quilting sutures and fibrin glue is one of the most common techniques used to tackle postoperative seromas. ${ }^{18}$ Although promising with regard to its efficiency in reducing postoperative seroma formation, the costs of fibrin glue ${ }^{19}$ and the added surgical time necessary for the quilting sutures ${ }^{20}$ are major drawbacks of this approach. Another tenet is based on preventing the degradation of already formed fibrin complexes around the vessels by fibrinolytically active components of the plasma. By inhibiting fibrin degradation, additional accumulation of exudate can be prevented. For example, tranexamic acid, an antifibrinolytic agent, has been tested in a randomized controlled trial but failed to show any statistically significant difference, ${ }^{15}$ despite the promising concept.

Nanotechnology and nanoparticle (NP) engineering offer numerous new opportunities for clinics. The small dimensions of the nanoparticles, as well as their controllable surface characteristics, can be harnessed for medical purposes. ${ }^{21}$ Leibler et al. $^{22}$ have recently introduced the concept of nano-bridging based on tissue adhesion on the large external surface area of inorganic silica and iron oxide nanoparticles, which then leads to macroscopic tissue gluing. They showed that the nano-bridging phenomenon can be harnessed to achieve tissue adhesion and strong closure of deep wounds in the skin and liver. More recently, the concept of nano-bridging has been extended from inert nanoparticles to nanomaterials exhibiting potent bioactivity, including bioglass/cerium oxidebased hybrid nanoparticles. ${ }^{23}$ In a recent study, ${ }^{24}$ we showed that the inherent procoagulant, ${ }^{23}$ neo-angiogenic, and antiinflammatory properties of zinc-doped strontium-substituted bioglass/ceria nanoparticles lead to increased skin flap survival in a rat model. Therefore, the prospect of extending nanobridging to bioactive nanoparticles with procoagulant and tissue regenerative properties for the prevention of seroma is promising. These zinc-doped strontium-substituted bioglass/ ceria nanoparticles unify the angiogenic and soft tissue regeneration properties of bioglass ${ }^{25,26}$ with endothelial cell proliferation and vascular sprouting induction, as well as antioxidant properties of ceria. ${ }^{23,27}$ Although both zinc ${ }^{28,29}$ and strontium $^{30,31}$ have angiogenic properties, zinc additionally 


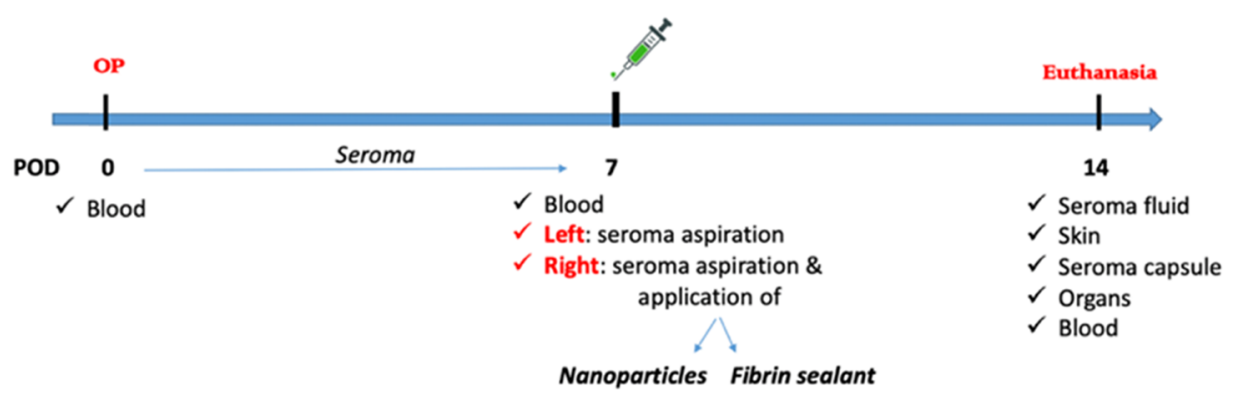

Figure 2. Experiment timeline. The operation was performed on postoperative day (POD) 0. On the same day, blood samples were collected. After 7 days, the rats developed seromas on both of the operated sides. The left side was used as control and the seroma fluid was aspirated, while the right side was used as treatment: the seroma fluid was aspirated and either nanoparticles or fibrin glue was injected and massaged in the cavity. Blood was also collected on this day. Euthanasia was performed on POD 14, when the seromas were aspirated, and various samples were collected for analysis.

exhibits anti-inflammatory activity. ${ }^{32,33}$ Importantly, zincdoped strontium-substituted bioglass/ceria nanoparticles can be produced in a scalable and sterile high-temperature flame spray pyrolysis process, fulfilling many of the prerequisites for later clinical translation. ${ }^{34,35}$

This study aimed to assess the ability of bioactive inorganic nanoparticle-based formulations to reduce seroma formation in a rat model, in direct comparison to one of the most frequently used clinical treatment and the current gold standard, fibrin glue.

\section{MATERIALS AND METHODS}

Nanoparticle Synthesis. The zinc-doped strontium-substituted bioglass/ceria nanoparticles (NPs) were produced by flame spray pyrolysis according to previously described methods. ${ }^{23,24}$ In short, calcium 2-ethylhexanoate, sodium 2-ethylhexanoate, tributyl phosphate, hexamethyldisiloxane, strontium acetylacetonate hydrate, Ce-2ethylhexanoate, and zinc acetylacetonate were diluted in tetrahydrofuran (THF) and sprayed through a nozzle. The created droplets were ignited by a flame and the nanoparticles were collected downstream after a nucleation process.

Nanoparticle suspension was prepared by ultrasonication immediately before application, at $20 \%$ amplitude for $4 \mathrm{~min}$, with a $70 \%$ power cycle (device: Sonopuls HD 4020, Bandelin electronic GmbH \& Co. KG, Berlin. Set for volumes from 20 to $900 \mathrm{~mL}$; ultrasonic nominal output max. $200 \mathrm{~W}$, ultrasonic generator GM 4200, ultrasonic converter UW 200, booster horn SH 200 G, and titanium flat tip TT 213, diameter $13 \mathrm{~mm}$ ).

Nanoparticle Characterization. Nanoparticle characterization was performed similarly as described in a previous study. ${ }^{36}$ Scanning transmission electron micrographs and energy-dispersive X-ray spectroscopy (EDS) elemental distribution maps of the as-prepared nanoparticles were recorded on a Talos F200X transmission electron microscope (TEM, Super-X EDS, four detector configuration, FEI) with an accelerating voltage of $200 \mathrm{kV}$. X-ray diffraction measurements were performed with a Bruker D8 advance diffractometer (40 $\mathrm{kV}, 40 \mathrm{~mA}, \mathrm{Cu} \mathrm{K} \alpha$ radiation) at $2 q=10-70^{\circ}$. Dynamic light scattering (DLS) hydrodynamic size and $\zeta$ potential measurements were performed with a Zetasizer (Nano ZS90, Malvern Instruments). Raman spectra of the nanoparticles were measured on a WITec Alpha $300 \mathrm{R}$ confocal Raman microscope with a UHTS 300 Vis spectrometer and an Andor Newton EMCCD. Fourier transform infrared (FTIR) spectra were collected using a Varian 640-IR spectrometer.

For elemental analysis, the as-prepared nanoparticles were weighted into poly(tetrafluoroethylene) tubes and mixed with $6 \mathrm{~mL}$ of $65 \% \mathrm{HNO}_{3}$ p.a. (Merck) and $1 \mathrm{~mL}$ of $30 \% \mathrm{H}_{2} \mathrm{O}_{2}$ p.a. (Merck). The samples were subsequently digested in a microwave and elements were quantified by inductively coupled plasma optical emission spectrometry (ICP-OES, Agilent 5110, Santa Clara, CA). Owing to the high silicate content and thus incomplete $\mathrm{Si}$ dissolution, the $\mathrm{Si}$ percentage was underestimated in some samples. For these samples, the residual mass was assumed to be $\mathrm{Si}$ as well. Other elements did not show incomplete recovery rates.

Surgical Model. First, a seroma model was developed by combining the techniques reported in the literature. ${ }^{13,17,37-39}$ Starting from the model described by Kulber et al., ${ }^{17}$ a bilateral model was envisioned: an incision was placed in the posterior axillary line, the cutaneous maximus and latissimus dorsi muscles were excised, and additional axillary lymphadenectomy was undertaken. The undersurface of the skin flap was scraped with a scalpel as described by Chung et al. $^{13}$ After hemostasis was achieved, the skin was closed with interrupted mattress sutures (Figure 1). This model allowed us to have an intraindividual comparison, with one side being used as control, while on the contralateral side of the same animal the treatment (either the nanoparticles or fibrin glue) was applied 1 week postoperatively, after the seroma fluid was aspirated.

All surgeries were performed under continuous inhalation anesthesia. Buprenorphine $(50 \mu \mathrm{g} / \mathrm{kg})$ was administered subcutaneously as preemptive analgesia $30 \mathrm{~min}$ prior to anesthesia. Isoflurane at $5 \%$ with oxygen $(1 \mathrm{~L} / \mathrm{min})$ was used for the induction of anesthesia $(2-3 \mathrm{~min})$ in an induction chamber. Then, the animals were placed on maintenance anesthesia at $1-1.5 \%$ isoflurane with $0.6 \mathrm{~L} / \mathrm{min}$ oxygen. All of the rats were maintained at normal body temperature using thermal pads and treated with ophthalmic ointment to both eyes to prevent desiccation. They were all monitored (respiratory rate, temperature) until awake. After the operations, rats were kept in a warm environment using a heating pad for at least $1 \mathrm{~h}$. Buprenorphine $(50 \mu \mathrm{g} / \mathrm{kg})$ was administered as postoperative analgesia just before final skin closure and every $12 \mathrm{~h}$ until postoperative day (POD) 2 . Further doses of buprenorphine were given if animals showed pain. Pain or distress was monitored carefully and graded based on a specially designed score sheet every day until the end of the experiment (Table S1). This study was conducted according to the ARRIVE guidelines ${ }^{40}$ and was approved by the Cantonal Animal Ethics Committee for Animal Experimentation, Bern, Switzerland (approval number BE 39/17).

Treatment Groups. Twenty Lewis rats weighing between 200 and $250 \mathrm{~g}$ were randomly assigned to two groups: 10 rats received the NPs as treatment, while the other 10 rats underwent treatment with fibrin glue (Tisseel, Baxter AG, 8152 Opfikon, Switzerland). The exact timeline of the study is depicted in Figure 2. Briefly, the rats were left for 7 days to develop seromas. The left side was used as a control and the seroma fluid was aspirated, while the right side underwent injection of either NP or fibrin glue after the aspiration of the seroma fluid. The NP suspension contained $15 \mathrm{mg}$ of nanoparticles $/ 1 \mathrm{~mL}$ of saline, while the same volume $(1 \mathrm{~mL})$ of fibrin glue was used for the other group. After another 7 days, the two sides of each rat were inspected and the seroma fluids were aspirated and collected where they were present. Moreover, ultrasound images were taken before aspiration to confirm the presence of seroma. Euthanasia was performed on POD 14, when harvesting of blood, 
(a)
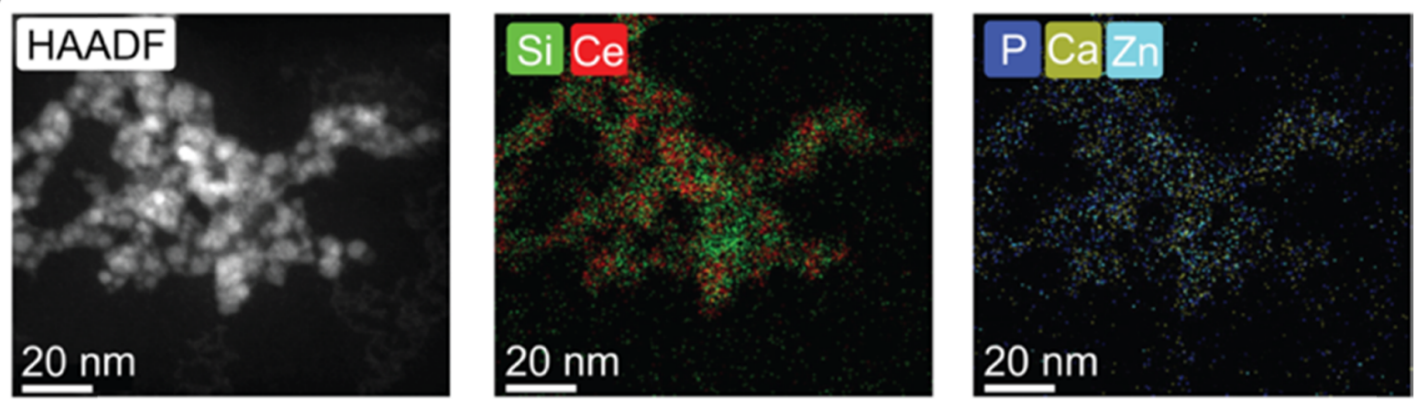

(b)

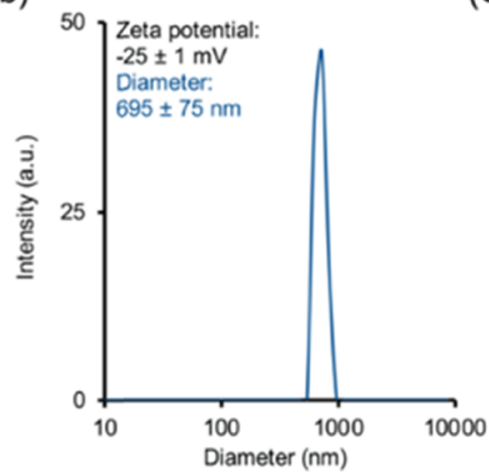

(c)

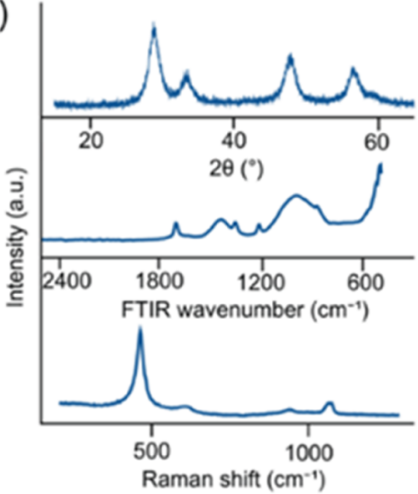

(d)

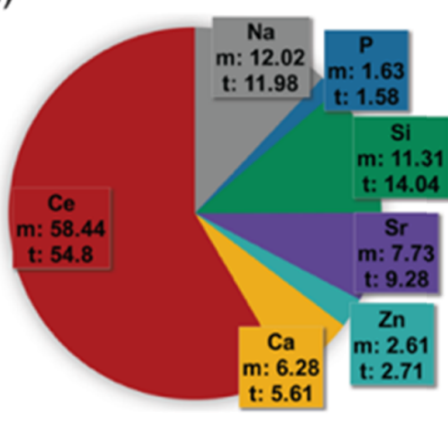

Figure 3. (a) High-angle annular dark-field (HAADF) electron micrographs of the nanoparticles including energy-dispersive X-ray spectroscopy mapping. (b) Dynamic light scattering results of the nanoparticles upon suspension in $10 \%$ phosphate-buffered saline (PBS) showing hydrodynamic diameter and $\zeta$ potential. (c) X-ray diffraction pattern showing characteristic ceria peaks. Fourier transform infrared (FTIR) spectrum with a pronounced broad peak at $1020 \mathrm{~cm}^{-1}$. Raman spectroscopy with a pronounced peak at $465 \mathrm{~cm}^{-1}$, which is a characteristic of ceria. (d) Elemental composition of the nanoparticles measured by inductively coupled plasma optical emission spectrometry (m: measured, $\mathrm{t}$ : theoretical).

seroma fluid (if present), seroma capsule, skin, and various organs for analysis was undertaken.

Cytokine Measurements. Protein extraction was performed as previously described by our group. ${ }^{24}$ Briefly, the M tubes (Miltenyi Biotec GmbH, Bergisch Gladbach, Germany) containing radioimmunoprecipitation assay buffer $(50 \mathrm{mM}$ Tris $-\mathrm{HCl}, \mathrm{pH} 8.0$, with $150 \mathrm{mM}$ sodium chloride, 1.0\% Igepal CA-630 (NP40), 0.5\% sodium deoxy cholate, and $0.1 \%$ sodium dodecyl sulfate) and protease inhibitor cocktail (Sigma) were used for skin samples, after being cut into small samples. The homogenates obtained with a gentle MACS Dissociator (Miltenyi Biotec $\mathrm{GmbH}$ ) were incubated on ice and underwent sonication. The supernatants underwent protein quantification, using a classical bicinchoninic acid assay. A bovine serum albumin standard $(2.0 \mathrm{mg} / \mathrm{mL}$ in $0.9 \%$ aqueous $\mathrm{NaCl}$ containing sodium azide, Thermo Fisher Scientific) was prepared and transferred to a flat transparent 96-well plate (Thermo Fisher Scientific). Luminex multiple assay (ProcartaPlex Mix\&Match Rat 6-Plex) was performed on tissue samples and plasma was collected on POD 0, 7, and 14 for the following proteins: vascular endothelial growth factor $\mathrm{A}$ (VEGF-A), interferon- $\gamma$ (IFN- $\gamma$ ), interleukin- $1 \beta$ (IL- $1 \beta)$, interleukin-4 (IL-4), interleukin-6 (IL-6), interleukin-10 (IL-10), monocyte chemoattractant protein-1 (MCP-1), and tumor necrosis factor- $\alpha$ $(\mathrm{TNF}-\alpha)$. The procedure was performed based on the original manufacturer's instruction manual, and plates were read on a FLEXMAP 3D system (Luminex, Austin, CA).

Systemic Effects. The aforementioned cytokines, together with creatinine, alanine aminotransferase (ALAT), and aspartate aminotransferase (ASAT), were analyzed from plasma collected on POD 0, 7, and 14.

Histological Analysis. Seroma capsules harvested on POD 14 underwent histological analysis after being stained with hematoxylin and eosin. Inflammation, fibrosis, granulation tissue, the presence of multinucleated giant cells, edema, congestion, and necrosis were determined and scored from 0 to 3, with 3 being the most severe.

Elemental Analysis of Organ Samples. Ceria content in the different rat organs was measured by transferring organ samples to a polytetrafluoroethylene container and mixing with $6 \mathrm{~mL}$ of $65 \%$ $\mathrm{HNO}_{3}$ p.a. (Merck) and $1 \mathrm{~mL}$ of $30 \% \mathrm{H}_{2} \mathrm{O}_{2}$ p.a. (Merck). The samples were then digested in a microwave and measured on an ICPOES instrument (Agilent 5110, Santa Clara, CA).

Statistical Analysis. GraphPad Prism 7 software (GraphPad Software Inc., La Jolla, CA) was used for statistical analysis. All values are expressed as mean \pm standard error or median with interquartile range, depending on the distribution of the data. The Shapiro-Wilk's test was used to assess data distribution. For the normally distributed data, comparisons within the same group between the control and the treatment sides, as well as between the same sides at POD 7 and POD 14 , were performed using the paired samples $t$ test. Comparisons between different groups were performed with the independent samples $t$ test and ordinary one-way analysis of variance (ANOVA). For the non-normally distributed data, Wilcoxon signed-rank test was employed for comparisons among the same group, while the MannWhitney $U$ test and the Kruskal-Wallis test were used for the between-groups analysis. $p$ values $<0.05$ were considered to indicate statistical significance.

\section{RESULTS AND DISCUSSION}

Inorganic Nanoparticle-Based Formulations Are Produced in a Controlled Manner. A bioactive nanoparticle formulation for topical application was prepared from flamemade mixed metal oxide NPs exhibiting properties optimized for wound healing purposes. The NPs were prepared by liquidfeed flame spray pyrolysis (LF-FSP) in one step from inexpensive liquid precursors. ${ }^{41}$ 

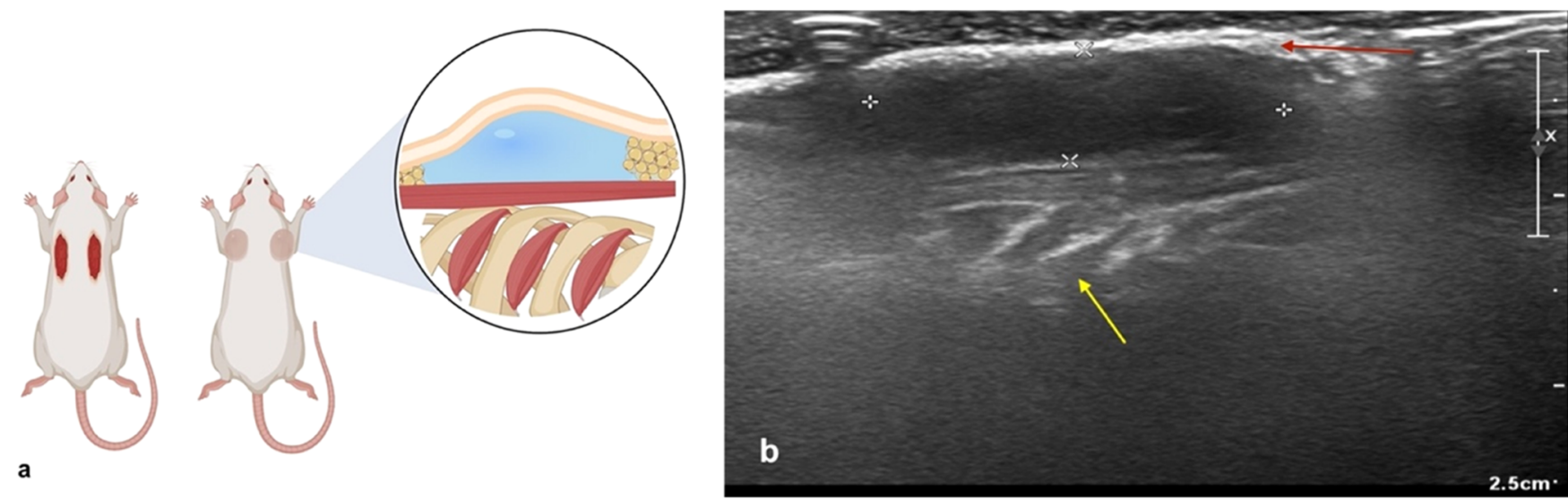

Figure 4. (a) Schematic representation of the seroma cavities on both sides of a rat, with a magnification of the sagittal plane through the lateral side, depicting skin, seroma cavity, muscle, and the thoracic cavity with the ribs and the intercostal muscles. (b) Representative ultrasound image depicting a seroma being delineated on the superior side by the skin (red arrow) and the inferior side by the muscle and thoracic cavity (yellow arrow). The seroma cavity has a length of $1.74 \mathrm{~cm}$ and a width of $0.5 \mathrm{~cm}$.

The physicochemical properties of the as-prepared zincdoped strontium-substituted bioglass/ceria nanoparticles are illustrated in Figure 3. Electron micrographs indicate a nanoparticle core size in the order of 5-10 nm (Figure 3a). Energy-dispersive X-ray spectroscopy mapping confirms the presence of $\mathrm{Ce}$, the $\mathrm{Zn}$ dopant, the elements contained in bioglass ( $\mathrm{Si}, \mathrm{Na}, \mathrm{Ca}, \mathrm{P}$ ), and the $\mathrm{Sr}$ substitution (Figure $3 \mathrm{a}$ ). Dynamic light scattering of the fractal-like particles in suspension gives an average hydrodynamic size of $695 \pm 75$ $\mathrm{nm}$ with a $\zeta$ potential of $-25 \pm 1 \mathrm{mV}$, which is comparable to similarly produced particles ${ }^{42}$ (Figure $3 \mathrm{~b}$ ). The powder X-ray diffraction pattern features the characteristic ceria peaks with the amorphous part of the bioglass masked by the high crystallinity of ceria (Figure 3c). On the FTIR spectrum, a broad peak around $1020 \mathrm{~cm}^{-1}$ is visible, which can be attributed to $\mathrm{Si}-\mathrm{O}-\mathrm{Si}$ bonds of the $\mathrm{SiO}_{4}$ tetrahedra in a bioglass framework. The sharp peak around $870 \mathrm{~cm}^{-1}$ is most likely a carbonate band that is characteristic of $\mathrm{CO}_{3}{ }^{2-}$ groups in carbonated apatites. The broad peak at $1450 \mathrm{~cm}^{-1}$ can be assigned to the $\mathrm{v}_{3}\left(\mathrm{CO}_{3}{ }^{2-}\right)$ band of carbonates adsorbed on the surface. ${ }^{43}$ The Raman spectrum shows the pronounced ceria peak at $460 \mathrm{~cm}^{-1}$. Inductively coupled plasma optical emission spectrometry of the nanoparticles demonstrates that their elemental composition is in excellent agreement with the theoretical values derived from the composition of their liquid precursor solutions (Figure 3d). Although flame-spraysynthesized nanoparticles yield process-intrinsic fractal agglomerates, which can only partially be broken up, the fractal-like characteristics (fractal dimension $D_{\mathrm{f}}$ of typically 1.9$2.3^{34,44,45}$ ) offer plenty of accessible surface area for proteins to adhere and interact giving rise to potent procoagulant properties and to some extent also tissue adhesive properties in the case of silica-containing (and especially bioglass) nanoparticles. ${ }^{23}$ The process robustness, scalability, and sterility offer an attractive route to large-scale production of metal oxide hybrid nanoparticles, especially for topical applications where hydrodynamic sizes are less critical.

Seroma Model Leads to Consistent Results. Several seroma models have been described in the literature. A seroma formation model in the rat after mastectomy was described in 1992 by Harada et al.; ${ }^{37}$ however, we consider the incision placed on the anterior half of the thorax too bothersome for the rats. Kulber et al. ${ }^{17}$ recorded a $90 \%$ rate of seroma formation after excising the latissimus dorsi muscle through an incision placed in the midaxillary line, while another group recorded a $74 \%$ seroma rate when excising the latissimus dorsi muscle and performing axillary lymphadenectomy through a posterior midline incision of the thorax. ${ }^{38}$ Alternatively, scraping the inner surface of the skin flap for lymphovascular disruption has also been described. ${ }^{13,39}$ Only the combination of the aforementioned techniques (i.e., the latissimus dorsi excision with lymphadenectomy and scraping of the inner surface of the flap) allowed us to obtain a $100 \%$ reliable rate of bilateral postoperative seroma, which then served as a model to investigate the treatment benefits of nanoparticle treatment (Figure 4). The serous fluid volumes ranged from 0.5 to $2 \mathrm{~mL}$, with minimal intraindividual left to right differences (mean 0.8 $\mathrm{mL}$, standard deviation (SD) $0.2 \mathrm{~mL}$ ). Moreover, the pain scores recorded throughout the experiments ranged from 0 to 2 , with scores of 1 and 2 present only on POD 1 and 2, when the rats received the usual postoperative analgesia. Afterward, analgesia was not deemed necessary, since all animals had a daily score of 0 until the end of the experiment.

Zinc-Doped Strontium-Substituted Bioglass/Ceria Nanoparticles Significantly Reduce Seroma Formation Compared to Fibrin Glues. Following the establishment of the seroma model, the experimental nanoparticle-based glue or fibrin adhesives were applied to treat seromas. Specifically, fibrin glue or the concentrated NP suspension was injected directly into the cavity (after aspiration of the seroma fluid), and the clinical progression during the experiment was followed. Seroma volume was measured on postoperative day (POD) 7 and POD 14 by aspiration. The nanoparticle-treated group did not show any volume on POD 14, translating to a $100 \%$ reduction of seroma. In contrast, the fibrin glue group still recorded seromas in 5 out of the 10 rats, with a seroma volume reduction of only 63\% (Figure 5a), which is in line with Kulber et al. ${ }^{17}$ who applied fibrin glue for seroma treatment in a similar fashion. When analyzing the untreated control side with the treatment side in the individual rats, we only reached a statistically significant reduction of seroma fluid in the NP group, which indicates a larger effect size (Figure $5 b)$. Despite some promising results in seroma reduction in experimental studies, ${ }^{17}$ fibrin glue has proven to be inferior, or even counterproductive ${ }^{46}$ in clinical studies. Moreover, the 


\section{Seroma formation}

a

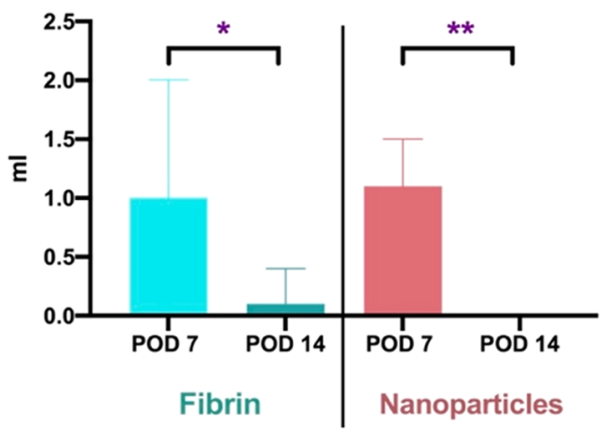

Seroma POD 14

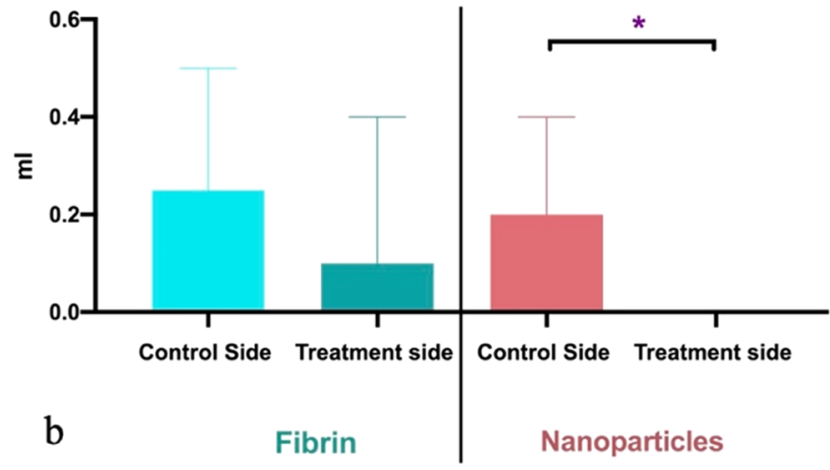

Figure 5. (a) Seroma formation reduction in the fibrin and nanoparticle groups between postoperative (POD) 7 and 14. On POD 7, seroma was aspirated on the treatment-designated side and either fibrin or nanoparticles were injected in the cavities. At POD 14, the amount of fluid was again quantified through aspiration. Data presented as median and range, $* p<0.05, * * p<0.01$, by Wilcoxon matched-pairs signed rank test. (b) Seroma formation in the fibrin and nanoparticle groups on POD 14-a comparison between the control side and the treatment-designated side. Data presented as median and range, $* p<0.05$, by Mann-Whitney test.

costs associated with its use are extremely high for a minor or nonexistent reduction in seroma formation. ${ }^{19}$

Although the main principle of reducing seroma focuses on the anti-inflammatory effects of the substances used, it is important not to compromise the wound healing process. In our study, we did not record any wound healing problems, in contrast to reported treatments with triamcinolone acetonide or other glucocorticoid-like substances. ${ }^{39}$ Such drugs might be suitable for the reduction of seromas; however, the possible complications associated with their use (e.g., wound infection, wound dehiscence) may preclude a more regular use in clinical practice. Note that the zinc-doped strontium-substituted bioglass/ceria nanoparticles also exhibit potent tissue regenerative properties, as recently demonstrated in a skin flap model. $^{47}$

Importantly, the rats did not record a pain score higher than 2 throughout the experiments, as assessed through the score sheet (Table S1). Additional analgesia was therefore not necessary, in contrast to studies using experimental treatments, such as OK-432. ${ }^{48}$ As opposed to the anti-inflammatory action of the nanoparticles, OK-432 is a sclerotherapeutic agent that induces inflammation and subsequent sclerosis of the dead space, causing pain, swelling, and redness at the injection site. ${ }^{48}$

Anti-Inflammatory and Neo-Angiogenic Effects of Nanoparticles on Biochemical Analysis Correlate with a
Reduction of Seroma Formation. The seroma capsule harvested from the nanoparticle group at the end of the experiment (POD 14) showed a statistically significant increase in VEGF-A, as well as a decrease in IFN- $\gamma$ (Figure 6 ). Since one of the theories behind seroma formation focuses on an exaggerated inflammatory response, ${ }^{10,11}$ the antiinflammatory effect of the NPs indicated by a mild downregulation of the pro-inflammatory cytokine IFN- $\gamma$ might hint at a possible mechanism. Similar anti-inflammatory and neoangiogenic effects of the zinc-doped strontium-substituted bioglass/ceria nanoparticles have been found in a perforator flap model, ${ }^{24}$ which further supports the proposed mechanism of action of the nanoparticles. VEGF-A is a potent angiogenic cytokine, stimulating endothelial cell proliferation. On the one hand, in the wound healing process, its expression is important and is mostly warranted by macrophages, representing an important cytokine, especially in the early stages. On the other hand, it also enhances vascular permeability, which might lead to the secretion of various plasma proteins and stimulation of the granulation tissue. ${ }^{49,50}$ This increase in permeability by VEGF might stimulate the accumulation of fluid ${ }^{6}$ and could therefore be counterproductive in the treatment of seromas. However, in our study, the slight upregulation of VEGF-A correlated with a reduction in seroma formation in the nanoparticle group.

Since there were no seromas in the nanoparticle group on POD 14, we were only able to analyze the seroma fluid of the fibrin group (Figure 7). All of the cytokines, apart from IL-6, showed increased levels on POD 14 in the treatment group compared to the contralateral control side. Additionally, IFN- $\gamma$, IL-1 $\beta$, IL-10, and MCP-1 were significantly increased. The main role of fibrin glue, a hemostatic agent, in minimizing seroma formation is based on its ability to seal the open lymphatic and blood vessels and to close the dead space, but there is only limited information available in the literature regarding its effects on inflammation. Although IFN- $\gamma$, IL- $1 \beta$, and MCP-1 are known for their pro-inflammatory activity, IL10 is an anti-inflammatory cytokine and its increase is an interesting finding. IL-6 is a cytokine that works both as proinflammatory and anti-inflammatory. Its anti-inflammatory properties are exerted through the downregulation of TNF- $\alpha$ and the upregulation of IL-10. Although our study did record an increase in IL-10, we did not see any reduction of TNF- $\alpha$ in the fibrin group, therefore rendering the interpretation of IL- 6 intricate, since overall, the fibrin glue seems to exert a proinflammatory effect.

No Significant Differences in the Histological Analysis of Seroma Capsule. A mild inflammatory response was seen in all of the samples, corresponding to postoperative changes. The lack of multinucleated giant cells confirmed the absence of foreign body reactions in both the nanoparticle and fibrin glue groups. Even though we could not identify any statistically significant difference between the groups when looking at the seroma capsule from the control side, the fibrin glue-treated side, and the nanoparticle-treated side, there was a trend of decreased inflammation in the nanoparticle group, with a mean score of $1.7 \pm 0.6$, while the fibrin group recorded a $2.1 \pm 0.8$ mean value.

Biodistribution by Elemental Analysis Shows no Clearance to Other Organs. To assess whether nanoparticles would be redistributed to other organs following topical application, elemental analysis on tissues was performed by inductively coupled plasma spectroscopy. Elemental ceria 

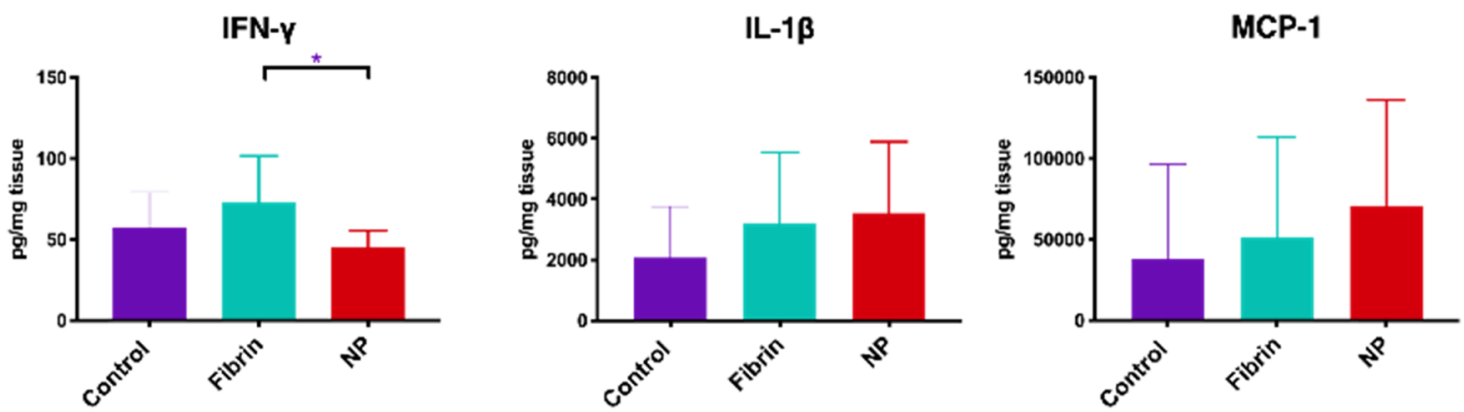

TNF-a
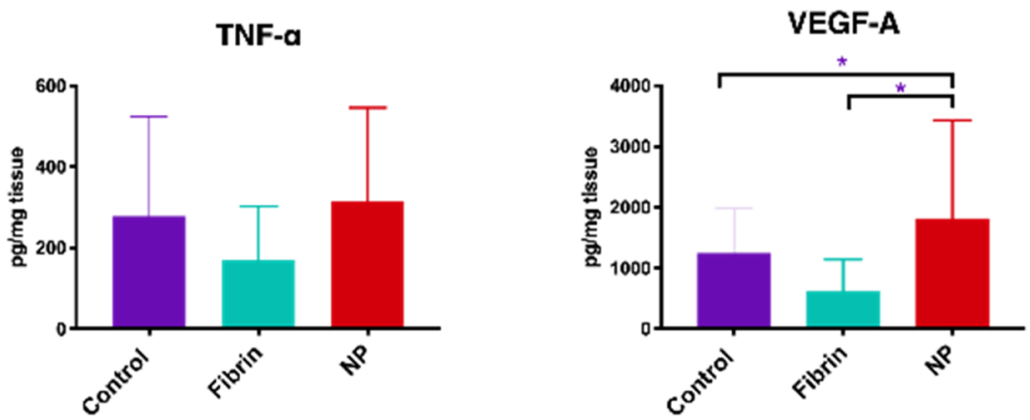

Figure 6. Biochemical analysis of the seroma capsule. The seroma capsule was harvested on postoperative day 14 from the control side (Control) and from the treatment sides in both groups (Fibrin: fibrin glue group; NP: nanoparticle group). Data presented as mean and SD, $* p<0.05$, by one-way ANOVA with Tukey's post hoc multi-comparisons test. (L-4, IL-6, and IL-10 were below the detection level).
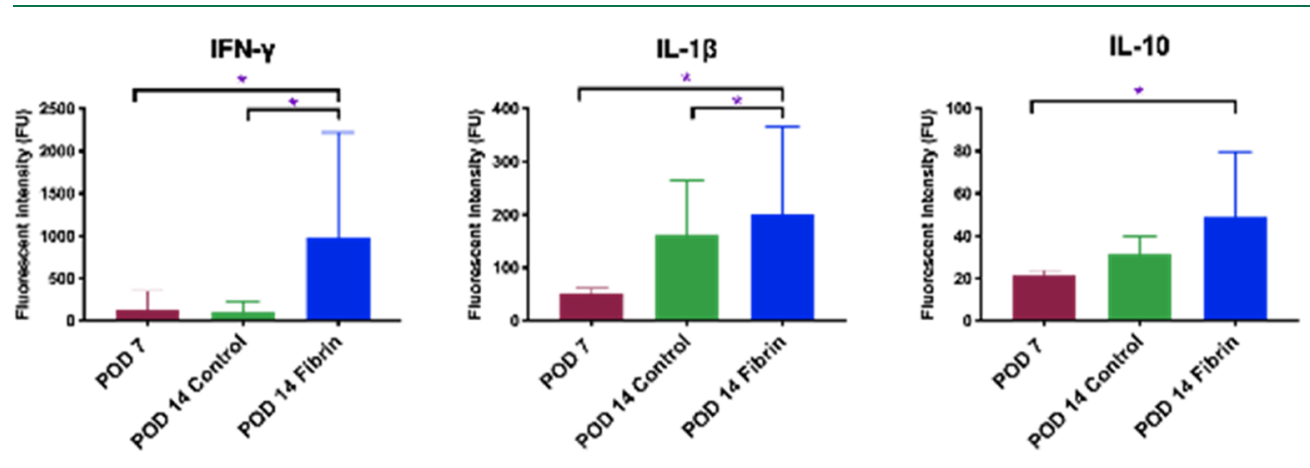

IL-4

IL-6

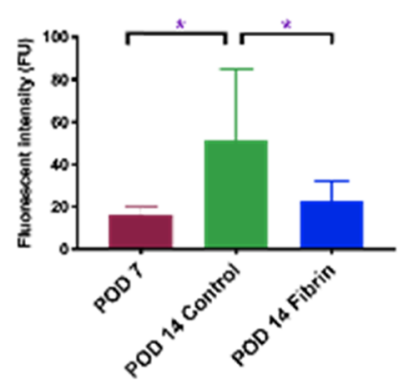

MCP-1

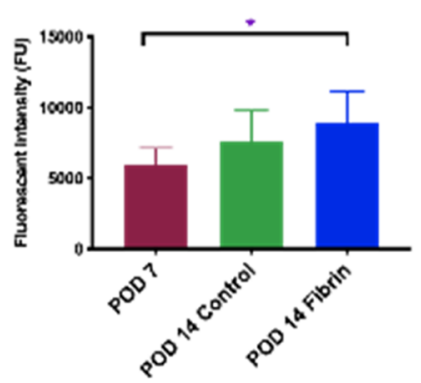

TNF-a

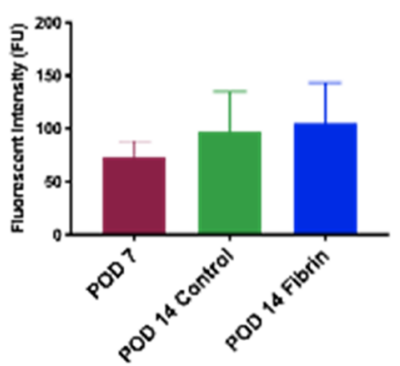

VEGF-A

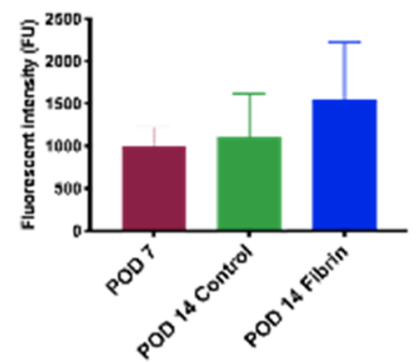

Figure 7. Biochemical analysis of seroma fluid in the fibrin group. The aspirated seroma fluid was collected on postoperative day (POD) 7 and POD 14. Although on POD 7 there was seroma fluid on both sides of the rats and in both groups, on POD 14 we only recorded seroma fluid on the control side of both groups (POD 14 Control) and on the treatment side of the fibrin glue group (POD 14 Fibrin). In the nanoparticle group, the seroma resolved on the treatment side on POD 14 and therefore we did not record any seroma fluid here. Data presented as mean and SD, $* p<$ 0.05 , by one-way ANOVA with Tukey's post hoc multi-comparisons test.

(Ce) was found to be a reliable tracer of such nanoparticles in tissue. ${ }^{36} \mathrm{Ce}$ values are standardized to express elemental Ce contents relative to organ weight in parts per million (ppm)
(Figure 8). The main finding is that there is no redistribution of the nanoparticles to the organs, indicating that the nanoparticles likely remain at the initial site of application 


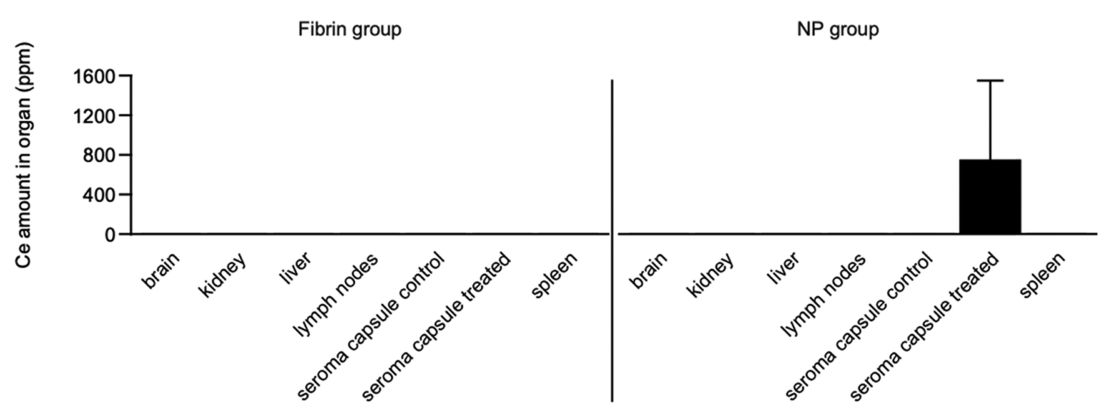

Figure 8. Ceria content in the organs and seroma capsule in the fibrin group and the nanoparticle group (NP group). Data presented as mean and SD.

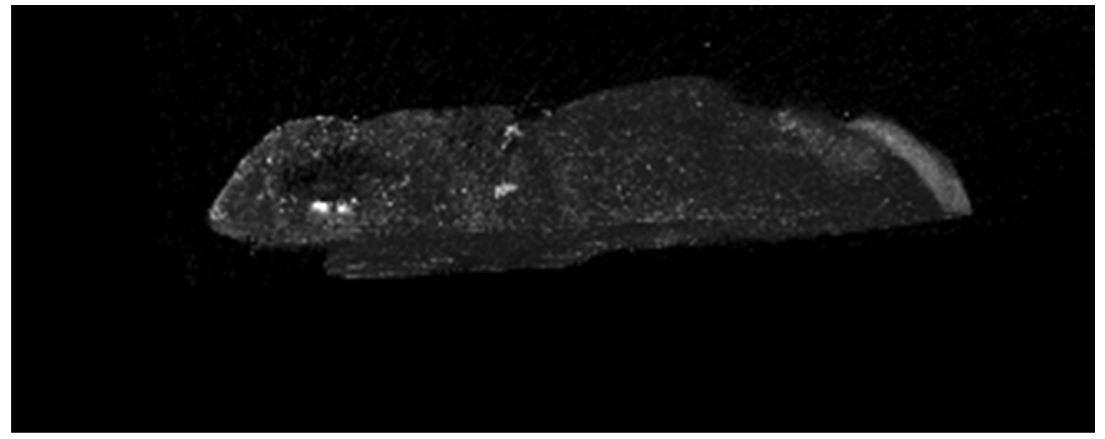

Figure 9. Microcomputed tomography (microCT). Skin capsule of the right treated side of a rat in the nanoparticle group: accumulations of particles visible.
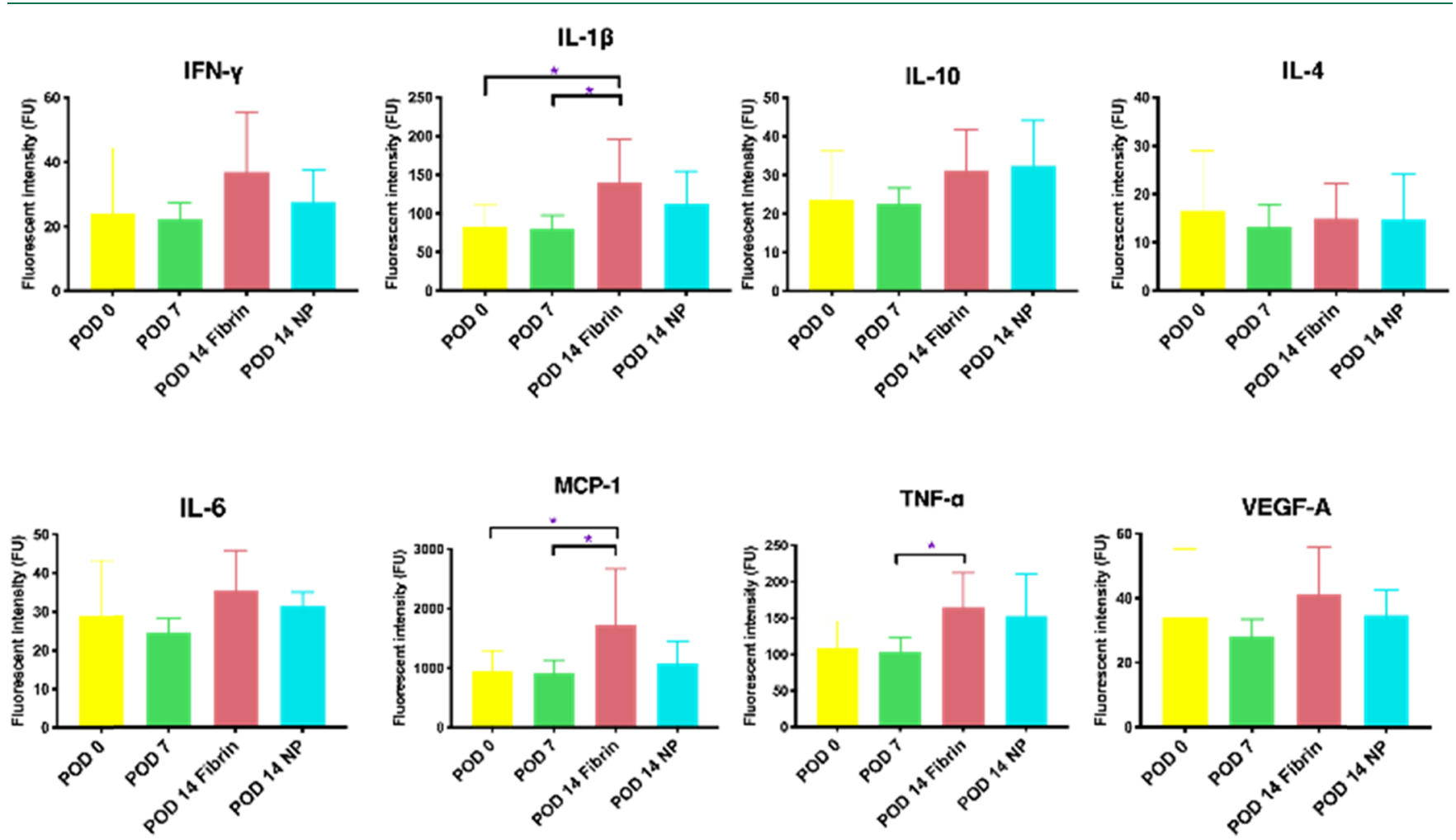

Figure 10. Plasma cytokine levels. Plasma analysis was undertaken at various time points: postoperative day (POD) 0 or operation day, POD 7 , and POD 14, in both the fibrin glue group and the nanoparticle (NP) group. Data presented as mean and SD, $* p<0.05$, by one-way ANOVA with Tukey's post hoc multi-comparisons test.

(skin and capsule of the seroma on the right side). Moreover, the Ce levels recorded in the organs remained at the background level found in fibrin-treated rats $(<0.01 \mathrm{ppm}$ $(\mu \mathrm{g} / \mathrm{L})$, which is the lower limit of detection). It is noteworthy, however, that in the case of 1 out of 10 rats, Ce levels above the noise were found in the liver, kidney, and spleen. This can likely be attributed to the entering of nanoparticles into a blood vessel during application. The risk and potentially 
associated side effects of such an unwanted event, although presumably rare, requires careful consideration and long-term studies. X-ray microtomography scans confirm the accumulation of nanoparticles within the right (treated) skin capsule (Figure 9). No indications of nanoparticle depositions were found in untreated skin capsules or lymph nodes.

Nanoparticle Treatment Has No Systemic Effect While Fibrin Glue Treatment Increases Systemic Expression of Inflammatory Cytokines. Importantly, systemic organ damage markers, such creatinine, ALAT, and ASAT plasma levels, remained unaltered during the experiments in all groups (Figure S1), with no differences recorded at POD 7 and 14 when compared to the values obtained at the beginning of the experiment (POD 0).

The plasma cytokine levels did not record any differences when comparing the results of the nanoparticle group at POD 14,7 , and 0 (baseline) (Figure 10), thus emphasizing the lack of systemic effects of the nanoparticle administration. However, for the fibrin group, we recorded a statistically significant increased inflammatory response: IL- $1 \beta$, MCP-1, and TNF- $\alpha$ had higher levels at POD 14 when compared to POD 7 and/or POD 0. These results highlight the fact that fibrin effects are not limited to the administration site, in contrast to the nanoparticle formulation, which seems to exclusively exert its anti-inflammatory and neo-angiogenic properties locally. Moreover, these findings are in line with the cytokine levels in the seroma fluid, which also showed mainly an upregulation of the pro-inflammatory cytokines in the fibrin group.

Limitations. Although the nanoparticles proved effective at reducing seroma formation, our study only evaluates the early postoperative period. Long-term studies would be necessary to determine whether this effect will last, or whether seromas could develop at a later stage. Moreover, even though we demonstrated the anti-inflammatory and neo-angiogenic effects of the nanoparticles through cytokine environment alterations, a more thorough understanding of the exact mechanism of the nanoparticles, and especially the risks associated with their use, is needed. In terms of formulation, the nanoparticles were dispersed in saline immediately before application. The development of a clinically friendlier application method would be worthwhile investigating to avoid the need for an ultrasonicator in the clinical setting that might not be so appealing to the clinicians. It is also noteworthy that while the nanoparticles can be produced from inexpensive precursors, the development will eventually drive the costs of a clinical nanoparticle product. Only a clinical study in direct comparison of the gold standard treatment based on fibrin glue will yield the clinical value and cost-effectiveness.

\section{CONCLUSIONS}

In summary, this work is a promising first example demonstrating the ability of inorganic nanoparticle-based formulations to reduce seroma formation in a newly developed, effective rat model. The application of the nanoparticle suspension 1 week after the surgery and after aspiration of the already formed seroma fluid translates into local attenuated inflammation and complete treatment of seroma, with no detectable systemic effects. Therefore, provided that these nanoparticle-based formulations are proven safe, the surgical field would benefit from the incorporation of these bioactive inorganic nanoparticles into the therapeutic management of seroma, a frequent postoperative complication.

\section{ASSOCIATED CONTENT}

\section{(s) Supporting Information}

The Supporting Information is available free of charge at https://pubs.acs.org/doi/10.1021/acsbiomaterials.1c00283.

Score sheet for postoperative pain evaluation and management (Table S1); creatinine, ALAT and ASAT levels in plasma (Figure S1) (PDF)

\section{AUTHOR INFORMATION}

\section{Corresponding Author}

Ioana Lese - Department of Plastic and Hand Surgery, Inselspital, Bern University Hospital, 3010 Bern, Switzerland; Email: ioana.lese@gmail.com

\section{Authors}

Catherine Tsai - Department for Biomedical Research, University of Bern, 3008 Bern, Switzerland

Martin Matter - Particles-Biology Interactions, Department of Materials Meet Life, Swiss Federal Laboratories for Materials Science and Technology (Empa), 9014 St. Gallen, Switzerland; Nanoparticle Systems Engineering Laboratory, Department of Mechanical and Process Engineering, ETH Zurich, 8092 Zurich, Switzerland

Tsering Wüthrich - Department for Biomedical Research, University of Bern, 3008 Bern, Switzerland

Helene Sophie Scheer - Department of Plastic and Hand Surgery, Inselspital, Bern University Hospital, 3010 Bern, Switzerland

Adriano Taddeo - Department for Biomedical Research, University of Bern, 3008 Bern, Switzerland

Mihai Adrian Constantinescu - Department of Plastic and Hand Surgery, Inselspital, Bern University Hospital, 3010 Bern, Switzerland

Inge Katrin Herrmann - Particles-Biology Interactions, Department of Materials Meet Life, Swiss Federal Laboratories for Materials Science and Technology (Empa), 9014 St. Gallen, Switzerland; Nanoparticle Systems Engineering Laboratory, Department of Mechanical and Process Engineering, ETH Zurich, 8092 Zurich, Switzerland

Radu Olariu - Department of Plastic and Hand Surgery, Inselspital, Bern University Hospital, 3010 Bern, Switzerland

Complete contact information is available at:

https://pubs.acs.org/10.1021/acsbiomaterials.1c00283

\section{Author Contributions}

I.L., M.A.C., I.K.H., and R.O. envisioned the design of the study. I.L., C.T., and T.W. performed the experiments and the collection of the data. I.L., C.T., M.M., T.W., H.S.S., and A.T. analyzed the samples and interpreted the results. M.A.C., I.K.H., and R.O. supervised and gave critical input to the project. I.L., M.T., I.K.H., and R.O. wrote the draft of the article, while all of the other authors reviewed the manuscript and gave their final acceptance for submission.

\section{Funding}

This work was partially funded by the Swiss National Science Foundation, SNSF Grant no. 181290.

\section{Notes}

The authors declare no competing financial interest. 


\section{ABBREVIATIONS}

ALAT, alanine aminotransferase; ASAT, aspartate aminotransferase; DLS, dynamic light scattering; EDS, energydispersive X-ray spectroscopy; FTIR, Fourier transform infrared; HAADF, high-angle annular dark-field; IFN- $\gamma$, interferon- $\gamma$; IL- $1 \beta$, interleukin- $1 \beta$; IL-4, interleukin-4; IL-6, interleukin-6; IL-10, interleukin-10; LF-FSP, liquid-feed flame spray pyrolysis; MCP-1, monocyte chemoattractant protein-1; NP, nanoparticle; NPs, zinc-doped strontium-substituted bioglass/ceria nanoparticles; POD, postoperative day; SD, standard deviation; THF, tetrahydrofuran; TNF- $\alpha$, tumor necrosis factor- $\alpha$; VEGF-A, vascular endothelial growth factor A

\section{REFERENCES}

(1) Marsh, D. J.; Fox, A.; Grobbelaar, A. O.; Chana, J. S. Abdominoplasty and Seroma: A Prospective Randomised Study Comparing Scalpel and Handheld Electrocautery Dissection. J. Plast., Reconstr. Aesthetic Surg. 2015, 68, 192-196.

(2) Rousseau, P.; Vincent, H.; Potier, B.; Arnaud, D.; Darsonval, V. Diathermocoagulation in Cutting Mode and Large Flap Dissection. Plast. Reconstr. Surg. 2011, 127, 2093-2098.

(3) Kumar, S.; Lal, B.; Misra, M. C. Post-Mastectomy Seroma: A New Look into the Aetiology of an Old Problem. J. R. Coll. Surg. Edinburgh 1995, 40, 292-294.

(4) Llewellyn-Bennett, R.; Greenwood, R.; Benson, J. R.; English, R.; Turner, J.; Rayter, Z.; Winters, Z. E. Randomized Clinical Trial on the Effect of Fibrin Sealant on Latissimus Dorsi Donor-Site Seroma Formation after Breast Reconstruction. Br. J. Surg. 2012, 99, 13811388.

(5) Delay, E.; Gounot, N.; Bouillot, A.; Zlatoff, P.; Rivoire, M. Autologous Latissimus Breast Reconstruction: A 3-Year Clinical Experience with 100 Patients. Plast. Reconstr. Surg. 1998, 102, 14611478.

(6) Kuroi, K.; Shimozuma, K.; Taguchi, T.; Imai, H.; Yamashiro, H.; Ohsumi, S.; Saito, S. Pathophysiology of Seroma in Breast Cancer. Breast Cancer 2005, 288-293.

(7) Aitken, D. R.; Minton, J. P. Complications Associated with Mastectomy. Surg. Clin. North Am. 1983, 63, 1331-1352.

(8) Tadych, K.; Donegan, W. L. Postmastectomy Seromas and Wound Drainage. Surg. Gynecol. Obstet. 1987, 165, 483-487.

(9) Purkayastha, J.; Hazarika, S.; Deo, S. V. S.; Kar, M.; Shukla, N. K. Post-Mastectomy Chylous Fistula: Anatomical and Clinical Implications. Clin. Anat. 2004, 17, 413-415.

(10) Watt-Boolsen, S.; Nielsen, V. B.; Jensen, J.; Bak, S. Postmastectomy Seroma. A Study of the Nature and Origin of Seroma after Mastectomy. Dan. Med. Bull. 1989, 36, 487-489.

(11) McCaul, J. A.; Aslaam, A.; Spooner, R. J.; Louden, I.; Cavanagh, T.; Purushotham, A. D. Aetiology of Seroma Formation in Patients Undergoing Surgery for Breast Cancer. Breast 2000, 9, 144-148.

(12) Qvamme, G.; Axelsson, C. K.; Lanng, C.; Mortensen, M.; Wegeberg, B.; Okholm, M.; Arpi, M. R.; Szecsi, P. B. Randomized Clinical Trial of Prevention of Seroma Formation after Mastectomy by Local Methylprednisolone Injection. Br. J. Surg. 2015, 102, 11951203.

(13) Chung, T. L.; Holton, L. H.; Goldberg, N. H.; Silverman, R. P. Seroma Prevention Using Mytilus edulis Protein in a Rat Mastectomy Model. Breast J. 2006, 12, 442-445.

(14) Gilbert, T. W.; Badylak, S. F.; Gusenoff, J.; Beckman, E. J.; Clower, D. M.; Daly, P.; Rubin, J. P. Lysine-Derived Urethane Surgical Adhesive Prevents Seroma Formation in a Canine Abdominoplasty Model. Plast. Reconstr. Surg. 2008, 122, 95-102.

(15) Oertli, D.; Laffer, U.; Haberthuer, F.; Kreuter, U.; Harder, F. Perioperative and Postoperative Tranexamic Acid Reduces the Local Wound Complication Rate after Surgery for Breast Cancer. Br. J. Surg. 2005, 81, 856-859.
(16) Bonnema, J.; Ligtenstein, D. A.; Wiggers, T.; Van Geel, A. N. The Composition of Serous Fluid after Axillary Dissection. Eur. J. Surg. 1999, 165, 9-13.

(17) Kulber, D. A.; Bacilious, N.; Peters, E. D.; Gayle, L. B.; Hoffman, L. The Use of Fibrin Sealant in the Prevention of Seromas. Plast. Reconstr. Surg. 1997, 99, 842-851.

(18) Sajid, M. S.; Betal, D.; Akhter, N.; Rapisarda, I. F.; Bonomi, R. Prevention of Postoperative Seroma-Related Morbidity by Quilting of Latissimus Dorsi Flap Donor Site: A Systematic Review. Clin. Breast Cancer 2011, 357-363.

(19) Azoury, S. C.; Rodriguez-Unda, N.; Soares, K. C.; Hicks, C. W.; Baltodano, P. A.; Poruk, K. E.; Hu, Q. L.; Cooney, C. M.; Cornell, P.; Burce, K.; Eckhauser, F. E. The Effect of TISSEEL Fibrin Sealant on Seroma Formation Following Complex Abdominal Wall Hernia Repair: A Single Institutional Review and Derived Cost Analysis. Hernia 2015, 19, 935-942.

(20) Pollock, T. A.; Pollock, H. Progressive Tension Sutures in Abdominoplasty: A Review of 597 Consecutive Cases. Aesthetic Surg. J. 2012, 32, 729-742.

(21) Hoshyar, N.; Gray, S.; Han, H.; Bao, G. The Effect of Nanoparticle Size on in Vivo Pharmacokinetics and Cellular Interaction. Nanomedicine 2016, 673-692.

(22) Rose, S.; Prevoteau, A.; Elzière, P.; Hourdet, D.; Marcellan, A.; Leibler, L. Nanoparticle Solutions as Adhesives for Gels and Biological Tissues. Nature 2014, 505, 382-385.

(23) Matter, M. T.; Starsich, F.; Galli, M.; Hilber, M.; Schlegel, A. A.; Bertazzo, S.; Pratsinis, S. E.; Herrmann, I. K. Developing a Tissue Glue by Engineering the Adhesive and Hemostatic Properties of Metal Oxide Nanoparticles. Nanoscale 2017, 9, 8418-8426.

(24) Lese, I.; Graf, D. A.; Tsai, C.; Taddeo, A.; Matter, M. T.; Constantinescu, M. A.; Herrmann, I. K.; Olariu, R. Bioactive Nanoparticle-Based Formulations Increase Survival Area of Perforator Flaps in a Rat Model. PLoS One 2018, 13, No. e0207802.

(25) Day, R. M. Bioactive Glass Stimulates the Secretion of Angiogenic Growth Factors and Angiogenesis in Vitro. Tissue Eng. 2005, 11, 768-777.

(26) Gorustovich, A. A.; Roether, J. A.; Boccaccini, A. R. Effect of Bioactive Glasses on Angiogenesis: A Review of in Vitro and in Vivo Evidences. Tissue Eng., Part B 2010, 199-207.

(27) Walkey, C.; Das, S.; Seal, S.; Erlichman, J.; Heckman, K.; Ghibelli, L.; Traversa, E.; McGinnis, J. F.; Self, W. T. Catalytic Properties and Biomedical Applications of Cerium Oxide Nanoparticles. Environ. Sci.: Nano 2015, 33-53.

(28) Yamaguchi, M. Role of Zinc in Bone Formation and Bone Resorption. J. Trace Elem. Exp. Med. 1998, 11, 119-135.

(29) Brandão-Neto, J.; Stefan, V.; Mendonça, B. B.; Bloise, W.; Castro, A. V. B. The Essential Role of Zinc in Growth. Nutr. Res. 1995, 15, 335-358.

(30) Marie, P. J.; Ammann, P.; Boivin, G.; Rey, C. Mechanisms of Action and Therapeutic Potential of Strontium in Bone. Calcif. Tissue Int. 2001, 121-129.

(31) Shahnazari, M.; Sharkey, N. A.; Fosmire, G. J.; Leach, R. M. Effects of Strontium on Bone Strength, Density, Volume, and Microarchitecture in Laying Hens. J. Bone Miner. Res. 2006, 21, $1696-1703$.

(32) Lang, C.; Murgia, C.; Leong, M.; Tan, L. W.; Perozzi, G.; Knight, D.; Ruffin, R.; Zalewski, P. Anti-Inflammatory Effects of Zinc and Alterations in Zinc Transporter MRNA in Mouse Models of Allergic Inflammation. Am. J. Physiol.: Lung Cell. Mol. Physiol. 2007, 292, L577.

(33) Hoppe, A.; Güldal, N. S.; Boccaccini, A. R. A Review of the Biological Response to Ionic Dissolution Products from Bioactive Glasses and Glass-Ceramics. Biomaterials 2011, 2757-2774.

(34) Starsich, F. H. L.; Herrmann, I. K.; Pratsinis, S. E. Nanoparticles for Biomedicine: Coagulation During Synthesis and Applications. Annu. Rev. Chem. Biomol. Eng. 2019, 10, 155-174.

(35) Gröhn, A. J.; Pratsinis, S. E.; Sánchez-Ferrer, A.; Mezzenga, R.; Wegner, K. Scale-up of Nanoparticle Synthesis by Flame Spray 
Pyrolysis: The High-Temperature Particle Residence Time. Ind. Eng. Chem. Res. 2014, 53, 10734-10742.

(36) Matter, M. T.; Li, J.; Lese, I.; Schreiner, C.; Bernard, L.; Scholder, O.; Hubeli, J.; Keevend, K.; Tsolaki, E.; Bertero, E.; Bertazzo, S.; Zboray, R.; Olariu, R.; Constantinescu, M. A.; Figi, R.; Herrmann, I. K. Multiscale Analysis of Metal Oxide Nanoparticles in Tissue: Insights into Biodistribution and Biotransformation. Adv. Sci. 2020, 7, No. 2000912.

(37) Harada, R. N.; Pressler, V. M.; McNamara, J. J. Fibrin Glue Reduces Seroma Formation in the Rat after Mastectomy. Surg. Gynecol. Obstet. 1992, 175, 450-454.

(38) Hurwitz, Z. M.; Ignotz, R. A.; Rowin, C.; Freniere, B. B.; Lalikos, J. F.; Dunn, R. M. Seroma Formation in Rat Latissimus Dorsi Resection in the Presence of Biologics: The Role of Quilting. Ann. Plast. Surg. 2015, 75, 338-342.

(39) Choi, M. S.; Kim, H. K.; Kim, W. S.; Bae, T. H.; Kim, M. K. A Comparison of Triamcinolone Acetonide and Fibrin Glue for Seroma Prevention in a Rat Mastectomy Model. Ann. Plast. Surg. 2012, 69, 209-212.

(40) Percie du Sert, N.; Hurst, V.; Ahluwalia, A.; Alam, S.; Avey, M. T.; Baker, M.; Browne, W. J.; Clark, A.; Cuthill, I. C.; Dirnagl, U.; Emerson, M.; Garner, P.; Holgate, S. T.; Howells, D. W.; Karp, N. A.; Lazic, S. E.; Lidster, K.; MacCallum, C. J.; Macleod, M.; Pearl, E. J.; Petersen, O. H.; Rawle, F.; Reynolds, P.; Rooney, K.; Sena, E. S.; Silberberg, S. D.; Steckler, T.; Würbel, H. The ARRIVE Guidelines 2.0: Updated Guidelines for Reporting Animal Research. PLoS Biol. 2020, 18, No. e3000410.

(41) Matter, M. T.; Furer, L. A.; Starsich, F. H. L.; Fortunato, G.; Pratsinis, S. E.; Herrmann, I. K. Engineering the Bioactivity of FlameMade Ceria and Ceria/Bioglass Hybrid Nanoparticles. ACS Appl. Mater. Interfaces 2019, 11, 2830-2839.

(42) Matter, M. T.; Furer, L. A.; Starsich, F. H. L.; Fortunato, G.; Pratsinis, S. E.; Herrmann, I. K. Engineering the Bioactivity of FlameMade Ceria and Ceria/Bioglass Hybrid Nanoparticles. ACS Appl. Mater. Interfaces 2019, 11, 2830-2839.

(43) MačKović, M.; Hoppe, A.; Detsch, R.; Mohn, D.; Stark, W. J.; Spiecker, E.; Boccaccini, A. R. Bioactive Glass (Type 45S5) Nanoparticles: In Vitro Reactivity on Nanoscale and Biocompatibility. J. Nanopart. Res. 2012, 14, No. 966.

(44) Tricoli, A.; Elmøe, T. D. Flame Spray Pyrolysis Synthesis and Aerosol Deposition of Nanoparticle Films. AIChE J. 2012, 58, 35783588 .

(45) Kelesidis, G. A.; Goudeli, E.; Pratsinis, S. E. Flame Synthesis of Functional Nanostructured Materials and Devices: Surface Growth and Aggregation. Proc. Combust. Inst. 2017, 36, 29-50.

(46) Akhtar, S.; Spyrou, G. E.; Fourie, L. R. Our Early Experience in the Use of Tissue Glue to Reduce the Incidence of Seroma Formation from the Latissimus Dorsi Flap Donor Site. Plast. Reconstr. Surg. 2005, 347-348.

(47) Lese, I.; Graf, D. A.; Tsai, C.; Taddeo, A.; Matter, M. T.; Constantinescu, M. A.; Herrmann, I. K.; Olariu, R. Bioactive Nanoparticle-Based Formulations Increase Survival Area of Perforator Flaps in a Rat Model. PLoS One 2018, 13, No. e0207802.

(48) Yang, Y.; et al. The Use of OK-432 to Prevent Seroma in Extended Latissimus Dorsi Flap Donor Site after Breast Reconstruction. J. Surg. Res. 2015, 193, 492-496.

(49) Howdieshell, T. R.; Callaway, D.; Webb, W. L.; Gaines, M. D.; Procter, C. D.; Sathyanarayana; Pollock, J. S.; Brock, T. L.; McNeil, P. L. Antibody Neutralization of Vascular Endothelial Growth Factor Inhibits Wound Granulation Tissue Formation. J. Surg. Res. 2001, 96, $173-182$.

(50) Esser, S.; Wolburg, K.; Wolburg, H.; Breier, G.; Kurzchalia, T.; Risau, W. Vascular Endothelial Growth Factor Induces Endothelial Fenestrations in Vitro. J. Cell Biol. 1998, 140, 947-959. 